\title{
An Efficient Hardware Implementation of CNN-Based Object Trackers for Real-Time Applications
}

\author{
Al-Hussein A. El-Shafie, Mohamed Zaki, and S. E. D. Habib, LSM IEEE
}

\begin{abstract}
The object tracking research continues to be active since long period because of the several real-world variations imposed in the tracking process, like occlusion, changing appearance, illumination changes and cluttered background. With wide range of applications, embedded implementations are typically pursed for the tracking systems. Although object trackers based on Convolution Neural Network (CNN) have achieved state-of-the-art performance, they challenge the embedded implementations because of slow speed and large memory requirement. In this paper, we address these limitations on the algorithm-side and the circuitside. On the algorithm side, we adopt interpolation schemes which can significantly reduce the processing time and the memory storage requirements. We also evaluate the approximation of the hardware-expensive computations aiming for an efficient hardware implementation. Moreover, we modified the online-training scheme in order to achieve a constant processing time across all video frames. On the circuit side, we developed a hardware accelerator of the online training stage. We avoid the transposed reading from the external memory to speed-up the data movement with no performance degradation. Our proposed hardware accelerator achieves 45.9 frames-per-second in training the fully connected layers.
\end{abstract}

Index Terms - object tracking, CNN, online training, deepfeature interpolation, hardware accelerator

\section{INTRODUCTION}

$\mathrm{T}$ HE object tracking field has wide application domains which are ever increasing like surveillance systems, intelligent robotics, unmanned vehicles and virtual reality.

Al-Hussein A. El-Shafie was with the Electronics and Communication Engineering Department, Faculty of Engineering, Cairo University, Giza, Egypt, as a PhD student. He has been working with several semiconductor companies (e-mail: elshafie_a@yahoo.com).

Mohamed Zaki is with the Computer Engineering Department, Faulty of Engineering, Al-Azhar University, Cairo, Egypt (e-mail: mzaki.azhar@gmail.com).

S. E. D. Habib is with the Electronics and Communication Engineering Department, Faculty of Engineering, Cairo University, Giza, Egypt (e-mail: serag@eng.cu.edu.eg).
The dramatic increase in the computational power has opened the door for the creation of new tracking algorithms to overcome the tracking field challenges [1].

Tracking algorithm traditionally employed hand-crafted features like pixel intensity, color and Histogram of Oriented Gradients (HOG) [2] to represent the target. However, hand-crafted features are not robust to sever appearance variations [3]. Deep features obtained from Convolution Neural Networks (CNN) achieved better performance than that obtained by hand-crafted features on recent tracking benchmarks. The adoption of CNNs in the tracking field can be classified into three use cases: First, CNNs are exploited to train the regression models of the Discriminative Correlation Filters (DCF) based trackers like in [4]-[6]. Second, a Siamese structure [7]-[9] is adopted where the two identical $\mathrm{CNN}$ branches are used to measure the similarity of two input patches. Third, fully-connected layers are added after the convolutional layers to score the input patches and classify them into object or background as in [3], [10]-[12]. All categories have achieved state-of-theart performance on the object tracking benchmarks and we focus on the third category in this paper.

Generally, real-time processing and small-form factor are desirable in most of the tracking applications. Hence, embedded platforms are the typical choice for implementing the tracking algorithms [1]. The implementation of the CNN-based object trackers on embedded platforms, however, confronts several challenges because of the slow speed, large memory requirements and the online training computations. For an efficient embedded implementation, the limitations of the CNN-based trackers need to be tackled while keeping acceptable performance levels. Initial results of our work were published in [13] where we showed the benefits of applying interpolation schemes to cut-down the required memory size in addition to speeding-up the processing time.

In this paper, we explore additional algorithm-level enhancements aiming for a hardware-efficient implementation, like dispensing the hard-negative mining step, approximating the softmax function and adopting a fixed dropout pattern. In addition, we spread the required 
processing of the online training stage across all the frames instead of performing the computations every specific frame intervals, and hence, a steady frames-per-second (fps) throughput can be achieved for the whole system.

Moreover, a fixed-point hardware accelerator is developed for the online-training computations. In general, $\mathrm{CNN}$ training requires at least $5 \mathrm{x}$ more multiply and accumulate (MAC) operations than the inference phase [14] with much larger amount of external memory accesses. These requirements challenge the real-time operation on embedded systems. In our work, we avoid the transposed reading from the external memory by re-forming the equations in the back-propagation path without impacting the performance. We also target the reduction of the access latency from the external memory via adopting efficient arrangement of the data storage and the access order.

This paper is organized as follows: Section II gives an overview of our proposed object tracker and the interpolation schemes employed. In section III, we evaluate additional algorithm-level features to simplify the hardware implementation. Section IV describes our proposed hardware accelerator for the online training. Experimental results and speed analysis for the complete tracker system are presented in section $\mathrm{V}$, and finally, section VI concludes our work.

\section{OVERVIEW OF OUR PROPOSED TRACKER}

We first presented our tracker named Interpolation and Localization Network (ILNET) in [12]. ILNET is based on the MDNET tracker [3], while it is modified to be suitable for embedded systems. The MDNET network consists of three convolutional layers, conv1:3, followed by three fully connected layers fc4:6 to classify the tested patches into object or background. In ILNET, the network is supplemented by fully-connected localization layers, fc7:9, in order to classify also the object location inside the patch into five positions: up, down, right, left and middle. The input of fc7 is the conv3 output, same input for fc4.

Generally, the required processing can be divided into three phases: tracking phase, training phase and network update. In the MDNET tracking phase, candidate patches are generated with Gaussian distribution around the object location in the previous frame. All these candidate patches are forwarded through the whole network and classified into object or background. The new predicted location can then be obtained by averaging the location of the candidate patches of the highest object score. In the training phase, training patches are generated around the new predicted location and the feature maps are extracted and stored every frame. The network update is carried-out either at fixed frame intervals, which is the long-term update, or when the object score drops severely during the tracking, which is the short term update as defined in [3].
The computation in the convolutional layers is the dominant part in the tracker operation. Therefore, the main purpose of our tracker is to reduce the number of the convolutional computations in the network and reuse the feature maps in the tracking phase and the training phase. In ILNET, the whole Region of Interest (ROI) is forwarded to the network instead of small random patches and a $15 \times 15 \times 512$ feature map is obtained. The object is represented by $3 \times 3 \times 512$ inside the ROI feature map. For the tracking phase, a coarse-fine localization scheme is adopted. A ROI feature map of size $15 \times 15 \times 512$ represents 169 candidates, each with feature map of size $3 \times 3 \times 512$. All the 169 candidates are classified into object or background, and then, the object patches are moved by the localization layers to obtain a coarse predicted location. For the fine localization, ILNET scores 100 fine samples around the coarse location. In order to achieve that, two feature maps of size $5 \times 5 \times 512$ are extracted around the coarse location at two scales, scale up and scale down. Bilinear interpolation is adopted to approximate the feature maps of the fine samples (100 samples) in the translation domain, and then linear interpolation is adopted to get the final feature map at the required fine scale. Fig. 1 illustrates the interpolation schemes employed in the fine localization step.

In the training phase, the ROI feature map obtained in the tracking phase in ILNET are reused to extract the feature maps of the positive and negative training patches by applying bilinear interpolation as well. Accordingly, the training patches are not forwarded through the convolutional layers, and hence, a significant reduction in the processing time is achieved. A speed improvement of $8.8 \mathrm{x}$ is obtained while achieving a comparable performance with the baseline tracker for all the tracing challenges.

Our overall tracking algorithm is presented in Algorithm 1. The convolutional layer weights, $\left[W_{l}: W_{3}\right]$, are initialized by the VGG-M [15] network model pertained on the ImageNet dataset, while the weights of fully connected layers, $\left[W_{4}: W_{6}\right]$ and $\left[W_{7}: W_{9}\right]$, are randomly initialized. Hence, our tracker is not offline-trained on any video dataset. At frame $i, \operatorname{fmap}_{1, i}$ is a conv3 feature map of size $15 \times 15 \times 512$, while fmap $_{2, i}$ and fmap $_{3, i}$ are feature maps of size $5 \times 5 \times 512$ at scale up and scale down respectively.

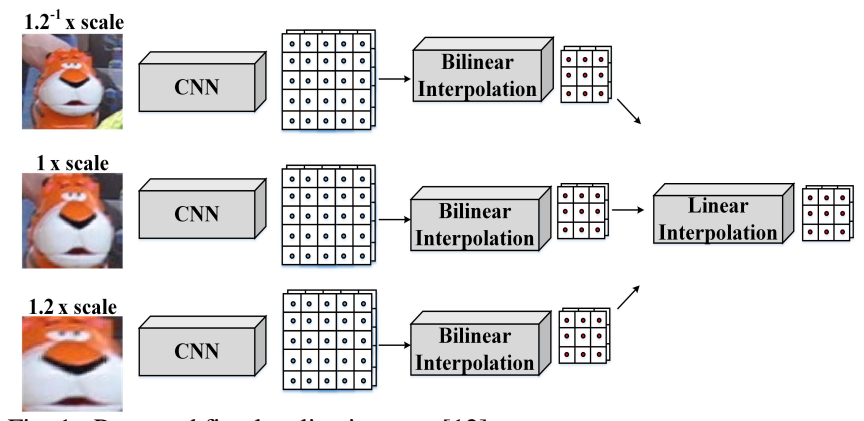

Fig. 1. Proposed fine localization step [12] 


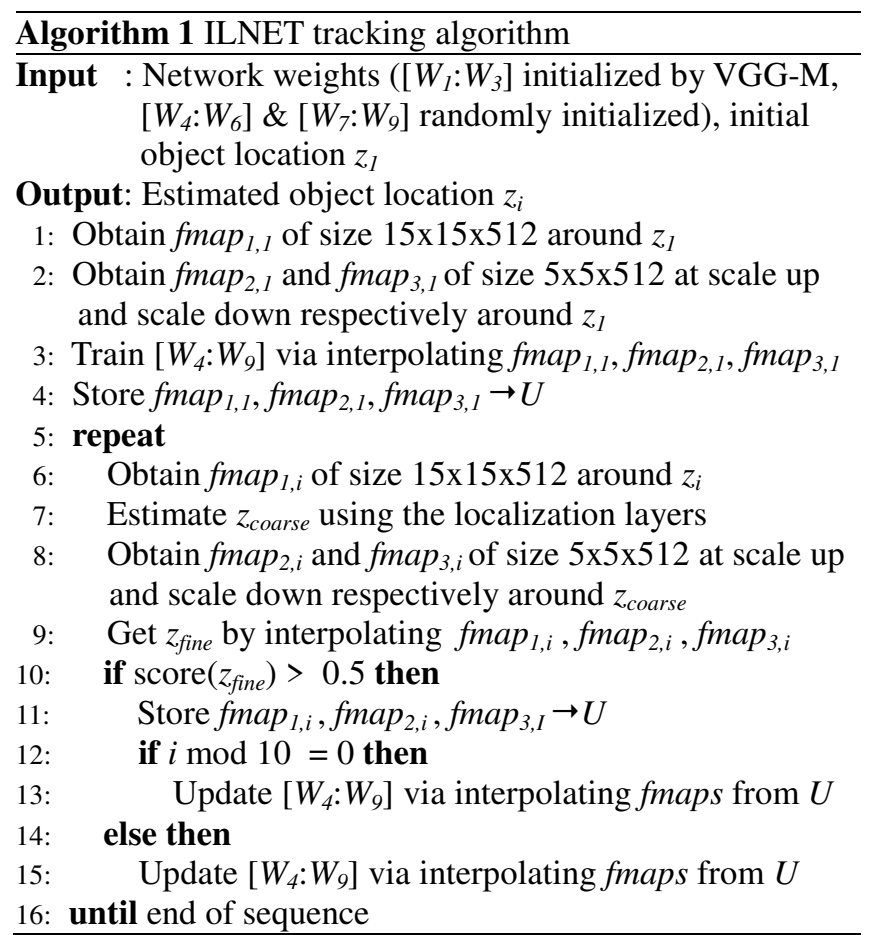

Moreover, our proposed tracker achieves a significant reduction in the memory storage requirement compared to the baseline tracker. Fig. 2 shows the breakdown of the required memory storage. The memory requirement is divided into weight storage, intermediate feature storage, fully connected training and conv 3 deep features which is used in the training. 16-bit fixed-point representation is assumed for all parameters. The localization layers used in ILNET adds an extra 5MB for the weight storage. In addition, the storage of the intermediate feature is slightly increased as well because we have increased the size of the input patches. Although there is an increase in the storage required for the weights and intermediate feature maps for our tracker, the proposed interpolation scheme allows a significant reduction in the storage of the conv3 feature maps.

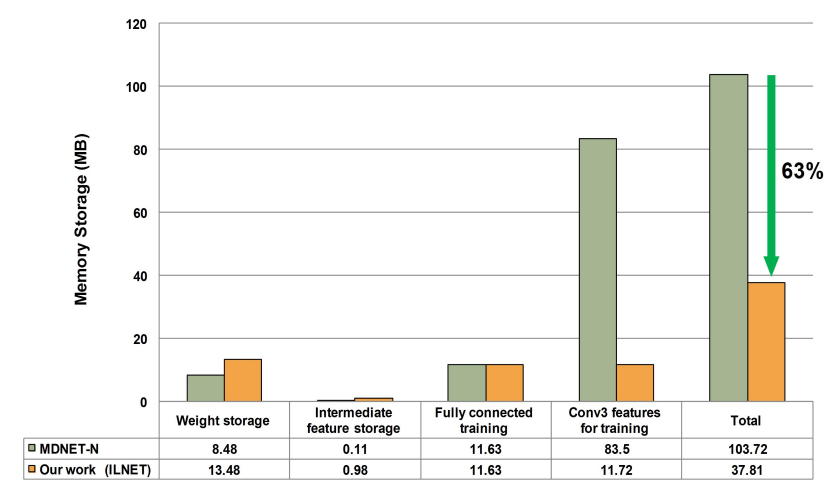

Fig. 2. Memory storage comparison between MDNET and ILNET
The long-term and short-term network update in both MDNET and ILNET are 100 and 20 frames respectively. The required memory storage for conv3 feature maps in MDNET is given by equations (1) and (2). conv3 feature maps are collected for 500 positive sample from the first frame, in addition to, 50 positive sample each frame in the long-period (100 frame) and 200 negative sample each frame in the short period (20 frame). For our tracker ILNET, there is no need to store the conv3 feature maps for all positive and negative samples each frame. As we exploit interpolation schemes, we can obtain and store a single and larger-size feature map each frame which can be reused to obtain the required feature maps when the training process needs it. We can store $5 \times 5 \times 512$ feature map at three scales for the positive training each frame in the long period (100 frame) and store $15 \times 15 \times 512$ feature map for the negative training each frame in the short period (20 frame). The required memory storage for conv3 feature maps in ILNET is given by equations (3) and (4). As shown in Fig. 2, our proposed tracker achieves a total memory reduction of $63 \%$.

$$
\begin{aligned}
\text { MDNET }_{\text {conv3 }}= & (\text { fmap size }) \times(\text { \# of fmap: } \text { initial frame }+ \\
& \text { positive long term }+ \text { negative } \text { short term })(1)
\end{aligned}
$$

$$
\begin{aligned}
\text { MDNET }_{\text {conv } 3} & =\underbrace{3 \times 3 \times 512 \times 2}_{\text {fmap size }} \times(\underbrace{500}_{\text {initial }}+\underbrace{50}_{\text {pos }} \times \underbrace{100}_{\text {long }}+\underbrace{200}_{\text {neg }} \times \underbrace{20}_{\text {short }}) \\
& =83.5 \mathrm{MB}
\end{aligned}
$$

$I L N E T_{\text {conv3 }}=$ positive fmap $\times$ long term $\times \#$ of scales + negative fmap $\times$ short term

$$
\begin{aligned}
\operatorname{ILNET}_{\text {conv3 }} & =\underbrace{5 \times 5 \times 512 \times 2}_{\text {pos fmap size }} \times \underbrace{100}_{\text {long }} \times \underbrace{3}_{\text {scale }}+\underbrace{15 \times 15 \times 512 \times 2}_{\text {neg fmap size }} \times \underbrace{20}_{\text {short }} \\
& =11.72 \mathrm{MB}
\end{aligned}
$$

\section{EVALUATION OF ALGORITHM-LEVEL FEATURES}

In this section, the algorithm-level features which have a severe impact on the hardware implementation are evaluated. We study the performance impact if we remove or approximate these features in addition to the gain that can be achieved in return. In [13], we explored the design space of the fixed-point representation of the main parameters of ILNET and we studied 11 ILNET variants using OTB-100 benchmark [16]. In this work, we evaluate the system-level features on ILNET5 which implements 8-bit for the weights and feature maps of the convolutional layers, and 16-bit for weights and feature maps of the fully connected layers.

Although we showed in [13] that the fixed-point represetation of the fully-connected layers can be reduced down to 13-bit and 12-bit for the weights and the feature maps respectively with small performance degaradation, we adopt 16-bit in this section because the byte-aligned 
representation simplifies the memory data allocation and data access.

In this analysis, we incremetaly study one sytem-feature at time and check the performance delta. We ran OTB-100 on all tracker variants and reported the performance and memory requirements as listed in table I.

\section{A. Removal of the multi-scales in the training}

In ILNET12, we removed the extraction of multi-scale feature maps which is employed in the training phase. The multi-scale feature maps were employed in the interplotion step to get the feature maps of the training patches. We depend on one scale only for the feature maps of the online training. Accordingly, the processing time and the required memory storage can be reduced, while trading-off a small performance degradation. Compared to ILNET5, the memory is reduced from 27.14 MB to $24.70 \mathrm{MB}$.

\section{B. Removal of the hard-negative mining}

The idea of hard-negative mining is to identify good negative samples for the training which is done via getting false positive classifications. In [3], 1024 negative samples are scored by the network, and then, the 96 negative samples with the highest positive score out of the 1024 tested samples are selected for the mini-batch training. The mini-batch consists of 32 and 96 positive and negative training samples respectively. Although hard-negative mining would enhance the classification capability of the tracker, it would challenge the hardware implementation. We would need to run the whole network for 1024 samples, score them and select-out the samples of the highest object scores to be used in the training phase. Therefore, in ILNET13, we evaluate the performance degradation if we remove the hard-negative mining step. It can be noticed that there is a considerable amount of performance loss in ILNET13 accordingly.

\section{Proposed online training scheme}

The online training step is carried out every tenth frame and with 10 iterations per update in MDNET and ILNET. This update scheme results in an inconsistent processing time across the frames. In addition to localization and collecting training samples each frame, the tenth frames would require longer processing time because of the online update process. We believe this update scheme is undesirable for the hardware implementation and it is important to achieve a constant processing times across all the frames. Therefore, we propose to modify the online update process where we perform the online training each frame and with a single iteration only. Consequently, the overall processing time would not change compared to the baseline tracker, while the processing time of the online training is spread evenly on the frames. The proposed update scheme is implemented in ILNET14 and the interesting point is that it has re-gained some of the performance loss caused by removing the hard-negative mining feature. Additionally, we made the Stochastic Gradient Descent (SGD) update much simpler by removing the momentum and decay update which would save $5 \mathrm{MB}$ required for the momentum storage. In ILNET14, the online update that we perform each frame is not actually affecting the filter weights directly. We store the updated weights temporarily and apply them to the network at the tenth frames. The required filter storage of the fully connected layers would double consequently, while removing the momentum storage compensates that and there will be no memory increase in total. We attempt to keep our proposed update scheme close to that of the baseline tracker. The difference between both approaches would be in the training patches adopted in the update process. For a batch of 10 frames, the baseline update scheme is carried out utilizing all the training patches collected from the last 10 frames as well as the previous frames. In our proposed update scheme, however, the update process is obviously carried out utilizing the training patches collected from the frames that are only received.

\section{Approximation for Softmax layer}

Softmax layer has been widely used in neural networks as an activation function and as a final claissification layer, while it involves expensive division and exponentiation finctions for the hardware implementation. Few works in literature [17]-[19] proposed efficient hardware implementaitons for the softamx function where all of them adopted domain transformation techinque. The domain transformaion is descibed in equations (5), (6) and (7).

$$
f\left(a_{i}\right)=\frac{e^{a_{i}-a_{\max }}}{\sum_{k=1}^{z} e^{a_{k}-a_{\max }}}
$$

$$
\ln \left(f\left(a_{i}\right)\right)=\left(a_{i}-a_{\max }\right)-\ln \left(\sum_{k=1}^{z} \exp \left(a_{k}-a_{\max }\right)\right)
$$

$f\left(a_{i}\right)=\exp \left(\left(a_{i}-a_{\max }\right)-\ln \left(\sum_{k=1}^{z} \exp \left(a_{k}-a_{\max }\right)\right)\right)$

It can be noticed from equation (7) that the division is removed and a logarithmic function is added. In [17], the logarithmic and expoentioal functions are implemened by Look-Up Tables (LUT). Linear fitting is adopted in [18] to approximate the logarithmic and expoenetaial functions to simpligy the hardware implementation. In [19], equation (7) is futher approximated to $f\left(a_{i}\right)=\exp \left(a_{i}-a_{\max }\right)$ in case of $\left(a_{i} \neq a_{\max }\right)$. 
TABLE I EXPLORATION OF SYSTEM-LEVEL FEATURES*

\begin{tabular}{|c|c|c|c|c|}
\hline Tracker & Success AUC & Precision & Memory (MB) & Feature outline \\
\hline ILNET5 & 0.606 & 0.843 & 27.14 & Baseline [13] \\
\hline ILNET12 & 0.599 & 0.832 & 24.70 & - Remove multi-scales in training \\
\hline ILNET13 & 0.583 & 0.819 & 24.70 & $\begin{array}{l}\text { - Remove multi-scales in training } \\
\text { - Remove hard-negative mining }\end{array}$ \\
\hline ILNET14 & 0.588 & 0.816 & 24.70 & $\begin{array}{l}\text { - Remove multi-scales in training } \\
\text { - Remove hard-negative mining } \\
\text { - Proposed training scheme (update every } 10 \text { frames) }\end{array}$ \\
\hline ILNET15 & 0.581 & 0.808 & 24.70 & $\begin{array}{l}\text { - Remove multi-scales in training } \\
\text { - Remove hard-negative mining } \\
\text { - Proposed training scheme (update every } 10 \text { frames) } \\
\text { - Softmax PWL }\end{array}$ \\
\hline ILNET16 & $\begin{array}{l}0.551^{1} \\
0.575^{2} \\
0.580^{3} \\
0.586^{4}\end{array}$ & $\begin{array}{l}0.757^{1} \\
0.791^{2} \\
0.800^{3} \\
0.809^{4}\end{array}$ & 24.70 & $\begin{array}{l}\text { - Remove multi-scales in training } \\
\text { - Remove hard-negative mining } \\
\text { - Proposed training scheme (update every } 10 \text { frames) } \\
\text { - Softmax PWL } \\
\text { - Dropout fixed patterns }\end{array}$ \\
\hline ILNET17 & 0.583 & 0.801 & 19.70 & $\begin{array}{l}\text { - Remove multi-scales in training } \\
\text { - Remove hard-negative mining } \\
\text { - Proposed training scheme (update every frame) } \\
\text { - Softmax PWL } \\
\text { - Dropout fixed pattern (30 pattern) }\end{array}$ \\
\hline
\end{tabular}

*For all ILNET variants given in this table, the weights and the feature maps are represented by 8-bit for the convolutional layers, and by 16-bit for the fully connected layers.

${ }^{1}$ No dropout, ${ }^{2}$ dropout pattern: $8,{ }^{3}$ dropout pattern: $18,{ }^{4}$ dropout pattern: 30

We can see that equation (7) has two exact expoential functions and one logarithmic function. In our work, we propose to adopt Piece Wise Linear (PWL) approximation for the exponential and logarithmic functions. We started from Matab to opt a suitable number of lines and the location of the breakpoints in addition to the proper fixed point representation of all the intermediate results. First for $\exp \left(a_{k}-a_{\text {max }}\right)$, we can notice that the operand $\left(a_{k}-a_{\max }\right)$ is always less than or equal zero which would simplify the approiximation. For the logarithmic function, the operand value $\left(\sum_{k=1}^{z} \exp \left(a_{k}-\right.\right.$ $\left.a_{\max }\right)$ ) would be range from 1 to $z$ ( $z$ is two for the classification network and five for the localization network). Conseuqently, the output from the logarithic function would range from 0 to $\sim 1.61$. For the final expoenetial function, it can be noticed that the operand $\left(\left(a_{i}-a_{\max }\right)-\right.$ $\left.\ln \left(\sum_{k=1}^{Z} \exp \left(a_{k}-a_{\max }\right)\right)\right)$ would be also less than zero like the first exponential, and hence, we can apply the same PWL for both exponential functions in eqaution (7). We adopted five linear pieces and four linear pieces for $\mathrm{PWL}_{\exp }$ and $\mathrm{PWL}_{\mathrm{ln}}$ respectively. Fig. 3 and fig. 4 plot the PWL function along with the exact functions. A small performance loss is observed when we apply the PWL approximation to the ILNET15 tracker as shown in Table I.

\section{E. Dropout layer}

We study the effect of the dropout layer on the traning process in ILNET16. Having a fully random dropout in hardware would complicate the design. Hence, we apply pseduo-random dropout patterns and evaluate the performance. We tested few variants where the pattern for the same dopout layer is repeated every 8, 18 and 30 iterations. The performance of no dropout is also evaluated. It can be noticed that there is a significant impact of the dropout layer on the perofmance. Removing the dropout layer has a sever performance degradation, while for the pseudo-random patterns, the performacnce is getting better as the pattern size increases.

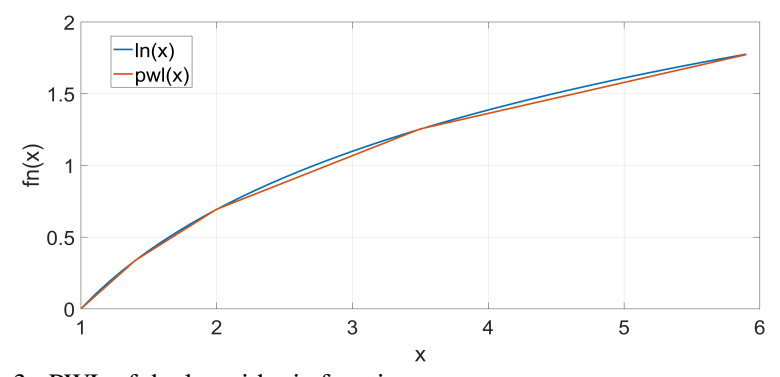

Fig. 3. PWL of the logarithmic function

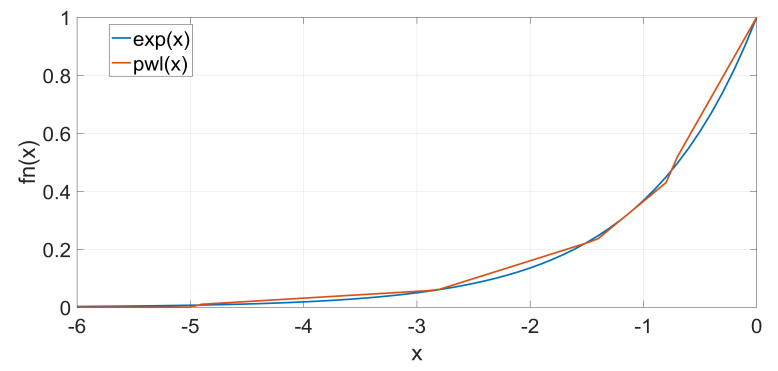

Fig. 4. PWL of the exponential function 


\section{F. Every-frame network update}

In ILNET17, we exteneded our porposed udpate scheme by applying the new filter weights directly to the network each frame. Hence, we would be able to get rid of the temporary filter storage and reduce the required memory storage. It can be noticed that performance is slightly affected compared to ILNET16 with a dropout pattern repeated every 30 iterations.

In addition to the advantages of simplyifing the hardware implementation and the memory reduction that are gained by applying the schemes in ILNET12 to ILNET17, a speed-up improvement of $1.6 \mathrm{x}$ is also obtained. The speed-up improvemnt arises mainly from removing the multi-scales in the training and from removing the hard-negative mining in ILNET12 and ILNET13 respectively. In addition, our proposed training scheme features a constant training time even in case of the frames that require a short-term update. We analyzed the speed of ILNET5 and ILNET17 trackers by running the Matlab system model on all OTB-100 videos using an Intel i7-3520M CPU system. ILNET5 runs in 0.9 second per frame, while ILNET17 run in 0.55 second per frame.

\section{ONLINE TRAINING HARDWARE ACCELERATOR}

Most of the previous publications on CNN hardware implementations focus on the inference phase and the convolutional layers. Generally, most CNN-based systems rely on offline training to obtain the filter weights which would be fixed throughout the inference phase of the network. As we mentioned, online training would slow-down the system speed and would require larger memory to store the online training dataset. Therefore, it is challenging to implement online training capability in embedded systems. However, online training is a key feature for our proposed tracker and other CNN-based trackers. We even dispense the offline training on any video dataset and solely depend on the online training in the initial frame and throughout the video frames. In this section, we present our proposed hardware accelerator for the online training of the fully connected layers.

We opted to develop and test our design on an FPGA as the target fabric, while our design is suitable for ASIC development as well. We target Xilinx VC709 evaluation board [20] which has Vertix-7 FPGA (XC7VX690T2FFG1761C) [21] and two 4GB DRAM3 modules. An important criterion to choose a suitable FPGA for our application is the number of DSP blocks available on the FPGA. In order to meet the target performance of CNN networks, a high-degree of parallel MAC operations has to be adopted. DSP blocks on FPGAs allow performing the MAC operations efficiently. The selected Vertix-7 FPGA has 3600 DSP slices where each slice contains a pre-adder, a $25 \times 18$ multiplier, an adder, and an accumulator. We think this number of DSP slices would be sufficient even if the whole tracker is implemented in hardware and still achieving the required real-time operation.

\section{A. Computation flow}

The common computation approach in the fully-connected layers is to store the weights and feature maps in the external memory and bring chunks of data to be processed on chip and write the results back to the external memory. There will be a trade-off between the data chunk size and the on-chip memory versus the number of external memory accesses. As the number of external memory accesses increases, the processing time and the energy consumption intuitively increase. Fig. 5 illustrates the main computation flow in the fully connected layers. The processing starts from $X_{4}$ which is the output from the convolutional layers or the stored feature maps. The size of $X_{4}$ is $128 \times 4608$ (128 is the mini-batch size and 4608 is the feature maps size, $3 \times 3 \times 512$ ). The fully connected layers $\mathrm{fc} 4$, fc5 and fc6 compute $X_{5}, X_{6}$ and $X_{7}$ respectively. The Softmax layer calculates the loss which is back-propagated in the network to get the delta weights: $d W_{6}, d W_{5}$ and $d W_{4}$, and the new propagated errors: $d X_{6}$ and $d X_{5}$. Matrix transpose operation has to be applied to $W_{6}, X_{6}, W_{5}, X_{5}$ and $X_{4}$ in the backward path. Hence, the main computation can be viewed as eight matrix multiplications: three in the forward path and five in the backward path as represented by yellow rectangles in fig. 5. They are big matrix multiplications with different sizes.

As matrix transpose is time-consuming, we re-form the matrix multiplications in the backward path by applying the transpose operator to both sides of the equations in the backward path. As shown in fig. 6, we store $\operatorname{loss}^{T}$ in the memory instead of loss and the back-propagated errors would be calculated in the transpose format as well, $d X_{6}^{T}$ and $d X_{5}^{T}$. In this proposed scheme, we would need to perform the transpose operation only when writing the loss and the delta weights. The external memory accesses required for writing the result in a transposed form is significantly less than that required for the transposed-reading to perform matrix multiplications. In addition, we will show that the weight update step can be done on the fly without requiring additional processing steps.

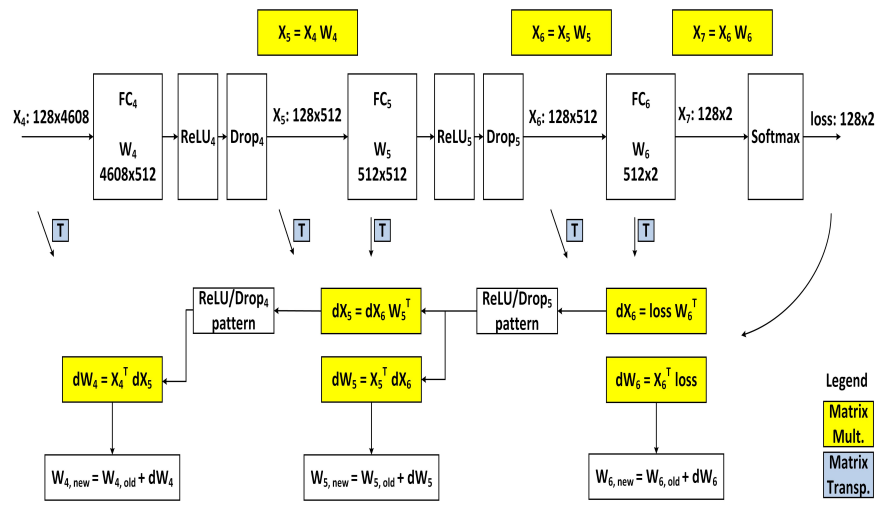

Fig. 5. The computation flow of a conventional online training 


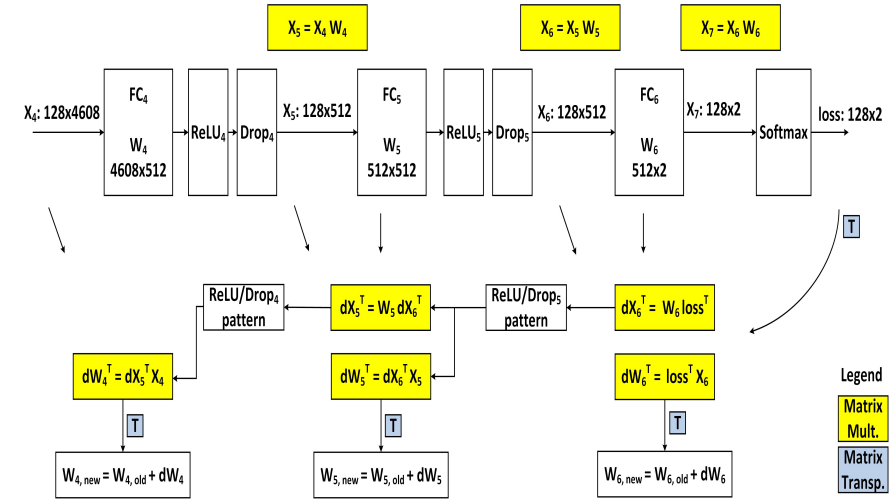

Fig. 6. The computation flow of the proposed online training

The arithmetic multiplier is a key element in this implementation. We can achieve better processing time by increasing the number of the parallel multiplications in the system. However, it is typically desired to reduce the number of multipliers to save the logic area. Moreover, the number of DSP elements in the FPGAs is limited. In order to get a sense of how many multipliers we need to achieve the target performance, we performed a quick analysis shown in Table II. We listed the number of the required multiplications in the forward path and backward path for the three layers and assuming $200 \mathrm{MHz}$ clock frequency and single update iteration. We can achieve $72.6 \mathrm{fps}$ with 256 multipliers. This performance should be acceptable such that when the fully connected computation is added to the convolutional layers computation, the whole system can achieve a real-time operation (i.e. around $30 \mathrm{fps}$ ). It is worth mentioning that this performance of $72.6 \mathrm{fps}$ is theoretical without taking into consideration the memory access latency or the Finite State Machine (FSM) delays.

TABLE II COMPUTATION BREAKDOWN IN FULLY CONNECTED LAYER

\begin{tabular}{|l|l|l|c|c|}
\hline \multirow{2}{*}{ FCx } & \multicolumn{2}{|c|}{ \# of multiplications } & \multicolumn{2}{c|}{ FPS } \\
& Forward & \multicolumn{1}{c|}{ Backward } \\
FC4 & $128 \times 4608 \times 512$ & 0 & 84.8 & \\
\cline { 1 - 4 } FC5 & $128 \times 512 \times 512$ & $\begin{array}{l}512 \times 512 \times 128+ \\
512 \times 128 \times 512\end{array}$ & 508.6 & \multirow{2}{*}{72.6} \\
\cline { 1 - 3 } FC6 & $128 \times 512 \times 2$ & $\begin{array}{l}512 \times 2 \times 128+ \\
2 \times 128 \times 512\end{array}$ & 130208.3 & \\
\hline
\end{tabular}

\section{B. Memory latency reduction}

Previous works have focused on reducing the number of the external memory accesses via transferring parts of the data to the on-chip buffer and maximizing the reuse of this on-chip data. Compression techniques have been also proposed to reduce the data size that is transferred from the external memory. Although these works result is a reduced number of memory accesses, they are sub-optimal as they do not consider the memory latency-per-access which also affects the energy consumption [22]. Therefore, in our work, we focus on reducing the external memory latency by adopting an efficient arrangement of storing and accessing the data.

First for the data storage, instead of storing the data matrix row-by-row or column-by-column, we store chunks of each row. The chunk is the maximum data that we can read from the DRAM in one clock cycle which is 512-bit (32 x 16-bit) on Xilinx VC709 evaluation board. Hence, starting from the first row, we store the first chunks ( 32 x 16-bit) of all the rows till we reach to the last row, then we store the second $(32 \times 16-$ bit) chunks of all the rows and so on. Fig. 7 illustrates the proposed order of data storage. If we manage to read and write the data in sequential addresses, this would yield the minimum memory latency.

Second for the memory access order and the matrix multiplication operation, we apply the same multiplication scheme for all the eight matrix multiplications where $[Z]=$ $[$ Mat 1$] *[$ Mat2]. Each matrix element is represented by $16-$ bit. We start by reading chunks (each chunk is 512 -bit $\equiv 32$ element) from eight rows of [Mat1] and store them on-chip. This read data can be considered a sub-matrix of size $8 \times 32$ elements, we call it [subMat1]. We then read a single chunk (32 elements) from [Mat2]. We perform 256 multiplications between the first column of [subMat1] and the 32 elements from [Mat2]. The partial sums are stored inside the registers of the MAC units and there is no need to transfer them to onchip memory or the DRAM. Then, the next chunk is read from [Mat2] and multiplied with the second column of [subMat1]. The second column of [subMat1] is already stored on chip and the partial sum is accumulated. A double-buffer is employed to store [subMat1] on-chip so that we maintain available data from [Mat1] when transitioning from the last column of [subMat1]. We continue the same sequence till reaching the chunk in the last row of [Mat2]. The output from the 256 multipliers will then give the first $8 \times 32$ elements of the final result $[Z]$ which are stored back to DRAM. We repeat the same sequence until we obtain all elements of $[Z]$.

It can be noticed that reading a single [subMat1] yields the minimum DRAM latency as the read addresses are sequential. The latency of filling the double-buffer by different submatrices whose DRAM start addresses are not sequential should not affect the processing time because filling the double-buffer is carried-out in parallel while performing the multiplications. The multiplications of all the 32 columns of a single [subMat1] would take at least 32 clock cycles which are typically sufficient to fetch another [subMat1] from the DRAM even if its start address is not contiguous with that of the previous sub-matrix.

For [Mat2], we read the first 32 element from all the rows sequentially. The MAC units perform the multiply and add operations on the fly while reading [Mat2] chunks till the last row is read. Hence, this scheme should also yield the minimum DRAM latency as all the read addresses are contiguous.

For the result $[Z]$, it is worth mentioning that it will be read in the following computation steps, and hence, it will be considered [Mat1] or [Mat2] of the new computation step. Therefore, we write the result in the same order we described before. We always obtain chucks of the result of size $8 \times 32$ and we write the chuck data in sequential addresses. 


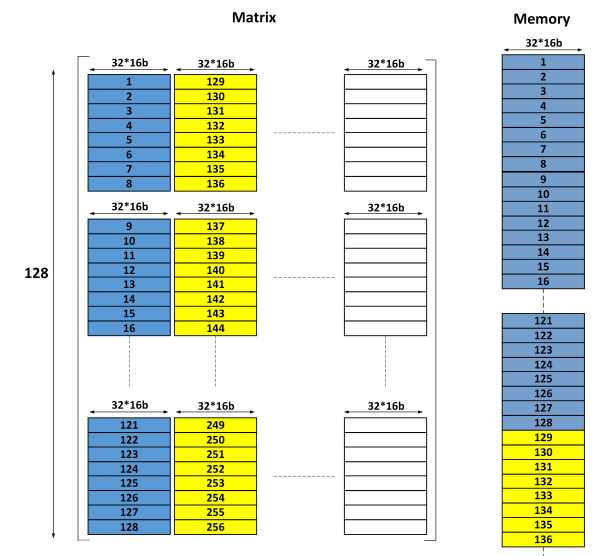

Fig. 7. Matrix storing order

\section{Weight update}

As we mentioned, we re-form the matrix multiplications in the backward path and obtain the transpose of the delta weights $\left[d w^{T}\right]$ in order not to calculate the transpose of [Mat2] and [Mat1]. For the weight update, if we opt to write the matrix $\left[d w^{T}\right]$ to DRAM, we would need to read it back in addition to the old weights then write back the updated weights. This scheme will negatively impact the processing speed. Instead, we do not write $\left[d w^{T}\right]$ directly to the DRAM. As we obtain chunks of $\left[d w^{T}\right]$ of size $8 \times 32$, we read chunks of $\left[W_{\text {old }}\right]$ of size $32 \times 8$. We update the weights and do the transpose on the fly. We then write chunks of $\left[W_{\text {new }}\right]$ of size $32 \times 8$ back to DRAM.

\section{ReLU and Dropout storage}

fc4 and fc5 are followed by ReLU and Dropout layers. ReLU layer saturates all negative values in the feature maps to zero and Dropout layer sets some values in the feature maps to zero randomly. We need to store the values that are set to zero in the forward path by ReLU and Dropout layers because they are required in the backward path when back-propagating the loss. Instead of storing the feature maps at each layer output (i.e. fc, ReLU and Dropout outputs), we store the output at the Dropout layer only in DRAM. We build a small array and store it on-chip memory where one bit per output neuron is employed to indicate whether this output neuron has been set to zero by ReLU, Dropout or not. As shown in Fig. 8, we store the pattern of ReLU and Dropout together which requires 8 $\mathrm{KB}(128 \times 512 \times 1$-bit) instead of $128 \mathrm{~KB}$ if we store the complete feature maps (128 x $512 \times 16$-bit). Fig. 9 shows an example of applying the ReLU and Dropout functions where we use a value of ' 1 ' to indicate that the neuron output is set to zero by either ReLU or Dropout layer.

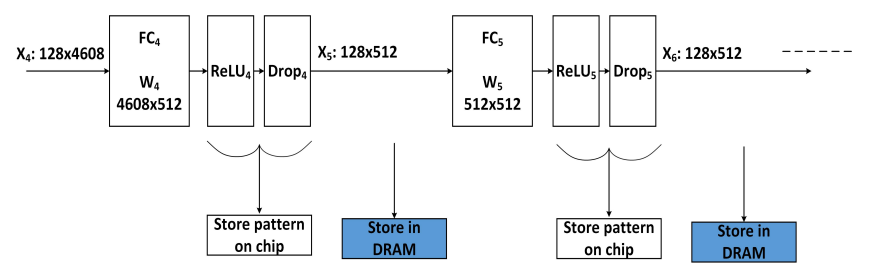

Fig. 8. ReLU and Dropout storage

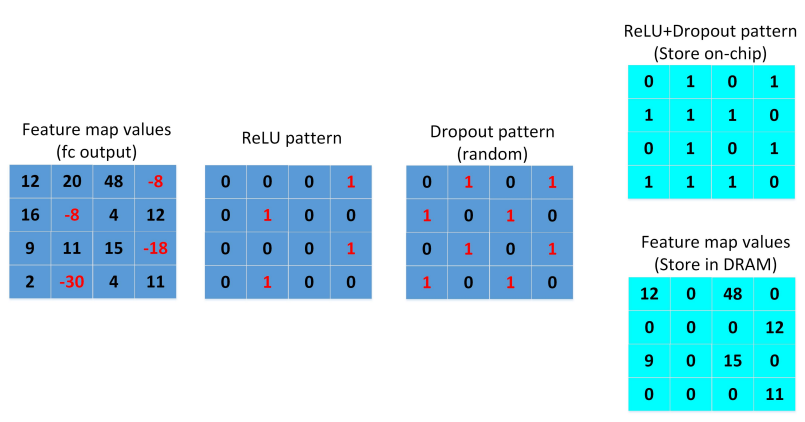

Fig. 9. Example of applying ReLU and Dropout patterns

\section{E. Memory allocation}

We store the feature maps in one DRAM and the fitter weights in the other DRAM. Our objective is to maximize the memory throughput in the system. Hence, we avoid reading [Mat1] and [Mat2] from the same DRAM. Therefore, we start by having $X 4$ in DRAM1, and all the weights: $W 4, W 5$ and $W 6$ in DRAM2. Then, we obtain and store $X 5$ and $X 6$ in DRAM1. After obtaining the loss, we store it in both memories because the loss would be required as [Mat1] when we calculate $\left[d W_{6}^{T}\right]$, while it would be required as [Mat2] when we calculate $\left[d X_{6}^{T}\right]$. We follow the same scheme after obtaining the propagated error $\left[d X_{6}^{T}\right]$, we write it in both memories because it would be considered [Mat1] and [Mat2] in the following computation steps. This writing scheme allows us to avoid reading [Mat1] and [Mat2] from the same DRAM in any of the computation steps.

\section{F. Top-level design description}

Fig. 10 shows the top-level block diagram of our proposed hardware design of the fully-connected layers. The blue arrows represent the data signals only and not the address or side-band signals. There are two FSMs to read [Mat1] and [Mat2] from the DRAMs. The maximum data size we can read from DRAM per clock cycle is 512-bit (32 elements each of 16-bit). As previously mentioned, the data for [Mat1] is stored in a double buffer of size $2 \times 8 \times 32 \times 16$-bit. There are 256 Processing Elements (PE) employed in our design which perform the MAC operations on two inputs. A bias would be added if required by the computation step. The data read from the double buffer is of size 8x16-bit, and are then replicated 32 times to generate input $A$ (256x16-bit) of the MAC units. Input $B$ (256x16-bit) of the MAC units comes from [Mat2] RD FSM after replicating the data eight times.

The output from the MAC units is stored in FlipFlops (FF) which will be written back to DRAM directly or processed first based on the computation step. In the softmax step, the MAC outputs are processed first by the softmax logic before writing to DRAM. In the weight update steps, the old weights are read by the weight RD FSM, got updated by the delta weights stored in the FFs and then written back to DRAM. We choose which DRAM to write in based on the computation step and the scheme we described previously. For the bias read and update, we store the bias values in chip RAM as they do not need large memory size. The bias is required for the 
matrix multiplication in the forward path. The bias update is carried out by the summation of the loss and the backpropagated errors.

The proposed design is described in Verilog with several parameterized parameters so that the same design can be ported easily to support other fully connected layers with different structures, input and weight sizes. The main parameters in our Verilog code are as follows:-

- a_total ,b_total: first and second dimension of [Mat1]

- $c_{-}$total , d_total: first and second dimension of [Mat2] $\left(b \_t o t a l=c \_t o t a l\right)$

- $a \_s t e p$ and $b \_s t e p$ : step size for reading [Mat1]

- $c \_s t e p$ and d_step: step size for reading [Mat2]

- memwr_step: step size for writing the result to DRAM

In addition, the number of the PEs and the associated interface is parameterized so that we can study the areaperformance tradeoff when increasing or decreasing the number of the PEs and choose the appropriate number based on the system needs.

There are also run-time configurations that would allow flexibility in the system when integrating the hardware accelerator with CPU. The start address of reading [Mat1] and [Mat2] from DRAM is configurable for all the computation steps and they should be provided by the CPU. In addition, the start address for writing the result to DRAM is configurable for all the computation steps. The selection of which DRAM to write the result in is also configurable. The CPU can also change the learning rate used by the accelerator at run-time based on the performance and system needs.

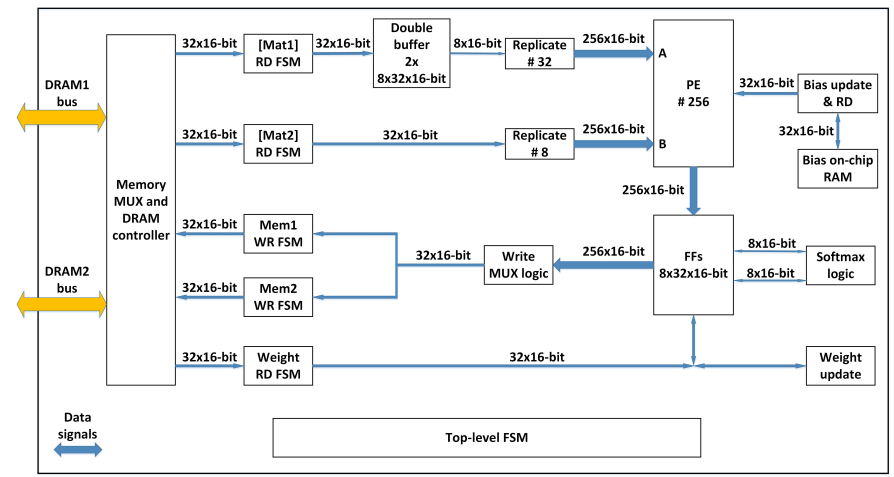

Fig. 10. Top-level block diagram of our proposed accelerator

\section{EXPERIMENTAL RESULTS AND ANALYSIS}

Test vectors are dumped from the Matlab model for all the design intermediate nodes. The same input vector and the initial weight values are used in simulating the design on the Register Transfer Level (RTL). The outputs at all the intermediate nodes from the hardware design are bit-matched with those of the Matlab model. We measured the design speed using an ideal memory model and a DRAM3 memory model. The ideal memory model has the same interface as that of DRAM3, while there are no wait states for read and write requests. Table III shows the performance of our design with the theoretical speed where there is no FSM or memory access overhead. It can be noticed that FSM overhead in our design is minimal where our design with the ideal memory model achieves pretty much the same performance as the theoretical case. For DRAM3 model, we achieved a memory access efficiency of $64.5 \%$ using our access scheme and the proposed order of the data storage.

TABLE III PERFORMANCE OF THE PROPOSED ACCELERATOR

\begin{tabular}{|l|l|}
\hline Design & $\begin{array}{l}\text { Performance at } 200 \mathrm{MHz} \text { and } \\
256 \text { multipliers }\end{array}$ \\
\hline Theoretical (No FSM, No memory) & $72.6 \mathrm{fps}$ \\
\hline Our design (Ideal memory model) & $71.2 \mathrm{fps}$ \\
\hline Our design (DRAM3 model) & $45.9 \mathrm{fps}$ \\
\hline
\end{tabular}

Our proposed design is synthesized and implemented by the Xilinx Vivado 2018.1 tool. We adopted the Xilinx generated design for the DRAM3 memory controllers. The FPGA resource utilization is shown in Table IV. In general, the utilization is low for all types of resources on the Vertix-7 FPGA. It is worth mentioning that the DRAM3 memory controller consumes $24.6 \%$ (22613 slices) of the whole LUT slices (91954 slices) used by our design.

Although our design is relatively small and Vertix-7 FPGA is relatively big in logic size, there was a congestion during placement and during routing which initially caused the Vivado tool not able to meet the required timing. For such congested designs, the Vivado tool would prioritize the routing completion of all signals over meeting the required timing. There are a couple of reasons for this congestion: the double buffer was implemented initially with BRAMs which may have limited the capability of the placer and router. High fan-out of several signals and big combinational logic for fixed-point conversion were reasons for the congestion as well. Therefore, we have implemented the double-buffer with registers (FFs) and added several pipelined FlipFlops to break long combinational paths. In addition, we have added several Multi Cycle Path (MCP) constraints for many signals. If intended by the design, adding MCP constraints on the failing paths would allow to meet the required timing for these paths without breaking the paths and adding more FlipFlops and latency. Moreover, we had to enable a special strategy for the Vivado implementation (Congestion_SpreadLogic_medium) which is recommended for highly congested designs. In some cases, there might be a need to explore the special strategy (Congestion_SpreadLogic_high) as well depending on the design complexity. After applying all these techniques, we are able to meet the required timing at $200 \mathrm{MHz}$.

TABLE IV RESOURCE UTILIZATION USING XILINX VERTIX-7

\begin{tabular}{|l|l|l|l|}
\hline Resource & Utilization & Available & Utilization \% \\
\hline LUT & 91954 & 433200 & 21.23 \\
\hline LUTRAM & 8637 & 174200 & 4.96 \\
\hline FF & 40338 & 866400 & 4.66 \\
\hline BRAM & 585 & 1470 & 16.25 \\
\hline DSP & 380 & 3600 & 44.71 \\
\hline IO & 6 & 850 & 18.75 \\
\hline MMCM & 3 & 20 & 15 \\
\hline PLL & 2 & 20 & 10 \\
\hline
\end{tabular}

Our HW accelerator can be adopted to train other MultiLayer Perception (MLP) neural networks that are employed in 
real-time applications on embedded devices. Some prior works [14], [23], [24] exist in literature to accelerate the training of the MLP or fully-connected neural networks. Approximate computing is adopted in [23] via inexact multipliers and bit-precision reduction to reduce the power consumption. The synapses that have lesser impact on the final error are obtained from the training phase and approximated by the inexact multipliers. In [24], the authors proposed a pipeline implementation of the Quasi-Newton method for training instead of the conventional batch training. The Quasi-Newton training method speeds-up the training convergence at the cost of memory resources.

A HW accelerator close to our work was recently published in [14]. The authors implement a HW accelerator in $65 \mathrm{~nm}$ ASIC to train the last fully connected layers in the MDNET based object tracker similar to our work. However, there are several differences. The whole network in [14] is trained offline on the VOT dataset. The authors added a new fullyconnected layer of weight size $1 \times 1 \times 512 \times 512$ in order not to train fc4 which has a weight size of $3 \times 3 \times 512 \times 512$. Hence, in the modified network, there will be four fully connected layers. fc5, fc6 and fc7 are only trained online, while the weights of fc4 are fixed. The idea behind this is to reduce the External Memory Access (EMA) where fc4 has the main contribution because of its large weight size. This modification in MDNET has negatively affected the performance by $5 \%$ on a Tracking Accuracy (TA) metric defined by the authors. In order to overcome the matrix transpose operation required in the error propagation and weight update steps, the authors employed Binary Feedback Alignment (BFA) where a constant matrix is used in the backpropagation path instead of the transposed weight matrix. BFA has performance degradation as well, reported to by $2.93 \%$ on TA. It is not mentioned, however, the actual impact of BFA and the scheme of not training $\mathrm{fc} 4$ online on the OTB benchmark. We believe it is important to have a unique metric, like OTB success AUC, across the different designs in order to achieve a fair comparison. The HW design in [14] implements a Run-Length Coding (RLC) to store the feature maps completely on-chip memory, while it brings chucks of the weights from the external memory to an on-chip memory of size $1 \mathrm{~KB}$.

On the other hand, in our proposed HW accelerator, we did not modify the fully-connected layers in order not to affect the performance. We still train fc4, fc5 and fc6 online. For the matrix transpose issue, we re-form the matrix multiplications in the backward path such that we do not need to read transposed weight and feature map matrices from the external memory. Instead, the propagated errors are calculated in the transposed form. Hence, we employ the normal backpropagation for the weight updates and avoid degrading the performance as what reported by [14] when using BFA. We store the feature maps and the propagated errors to the external memory which makes our design more scalable to larger-size networks. We have provided detailed OTB results on all the system-level features we studied. Table V shows a comparison summary of our work with [14].
As we target embedded systems, it is important to get the performance of the whole tracker operation including the inference and the training phases. We assume the $\mathrm{CNN}$ inference is carried out by the inference processor [25] which is the same inference processor assumed in [14]. We estimate the speed of the our tracker ILNET and MDNET when employing our training accelerator and the training accelerator in [14] respectively, while using the same inference processor for both. Table VI and Table VII show the maximum FPS that can be obtained when using the CNN processor [25] as the CNN inference for both MDNET and our proposed tracker ILNET respectively along with the computation breakdown. This analysis is carried out through obtaining the required MAC operations of the convolutional computations in both trackers. For MDNET, the number of MAC operations for one candidate is calculated and multiplied by the total number of candidates in the tracking phase and the training phase to get the total number of MAC operations. For ILNET, the number of MAC operations is calculated for each feature map size: one feature maps of size $15 \times 15 \times 512$ and two feature maps of size $5 \times 5 \times 512$. There are no convolutional computations required for the training phase. Accordingly, the CNN accelerator [25] can run ILNET convolution computations with $167.45 \mathrm{fps}$, while it can run MDNET convolution computations with $4.87 \mathrm{fps}$. It is evident that our modifications on the system-level of ILNET have achieved a significant improvement of the total tracker speed.

TABLE V COMPARISON SUMMARY

\begin{tabular}{|l|l|l|}
\hline & {$[14]$} & Our work \\
\hline Online-trained only & No & Yes \\
\hline Error propagation & BFA & Back-propagation \\
\hline EMA reduction scheme & RLC, BFA & No \\
\hline Implementation fabric & ASIC $65 \mathrm{~nm}$ & FPGA Vertix-7 \\
\hline Online training FPS & 16.2 FPS & 45.9 FPS \\
\hline
\end{tabular}

TABLE VI MDNET PERFORMANCE ON A CNN ACCELERATOR

\begin{tabular}{|l|l|}
\hline MDNET conv computation breakdown & value \\
\hline \# of conv MAC per one candidate & $121.664544 \times 10^{6}$ \\
\hline \# of runs in tracking phase & 256 \\
\hline \# of runs in training phase & 250 \\
\hline Total \# of MAC per frame & $61.562259264 \times 10^{9}$ \\
\hline Maximum throughput in [25] & $300 \mathrm{GOPS}$ \\
\hline Maximum inference FPS & $4.87 \mathrm{fps}$ \\
\hline MDNET tracker speed & $3.74 \mathrm{fps}$ \\
\hline
\end{tabular}

TABLE VII ILNET PERFORMANCE ON A CNN ACCELERATOR

\begin{tabular}{|l|l|}
\hline ILNET conv computation breakdown & Value \\
\hline \# of conv MAC to get a single $15 \times 15$ conv3 & $1323.007008 \times 10^{6}$ \\
\hline \# of conv MAC to get a single $5 \times 5$ conv3 & $231.079968 \times 10^{6}$ \\
\hline \# of runs in tracking phase & 1 for $15 \times 15$ conv3 \\
& 2 for $5 \times 5$ conv3 \\
\hline \# of runs in training phase & 0 \\
\hline \# of MAC in interpolation & $18.432 \times 10^{3}(4 \times 9 \times 512)$ \\
\hline \# of total MAC in interpolation per frame & $6.451200 \times 10^{6}$ \\
\hline Total \# of MAC per frame & $1.791618144 \times 10^{9}$ \\
\hline Maximum throughput in [25] & $300 \mathrm{GOPS}$ \\
\hline Maximum inference FPS & $167.45 \mathrm{fps}$ \\
\hline ILNET tracker speed & $36 \mathrm{fps}$ \\
\hline
\end{tabular}

\section{CONCLUSION}

We target an efficient implementation of CNN-based object trackers on embedded systems. In our previous works, we 
showed how interpolation schemes can be exploited to reduce the processing time and the memory requirements. In this paper, the impact of removing or approximating the systemlevel features that challenge the hardware implementation is evaluated. In addition, we propose a modified online training scheme where we spread the required training processing across the frames instead of carrying-out the training every specific frame interval, and hence, we can achieve a constant computation time for all the frames. On the circuit side, a fixed-point hardware accelerator for the online training of the fully connected layers is presented. We focused on reducing the access latency from the external memory via adopting an efficient arrangement of storing and accessing the data. We avoid the transposed reading from the external memory, which is a time-consuming operation, by re-forming the equations in the back-propagation path without affecting the tracker accuracy. Our proposed accelerator can train the fully connected layers at a rate of $45.9 \mathrm{fps}$ while operating at 200MHz.

\section{REFERENCES}

[1] A. H. A. El-Shafie and S. E. D. Habib, "Survey on hardware implementations of visual object trackers," IET Image Processing. 2019, doi: 10.1049/iet-ipr.2018.5952.

[2] N. Dalal and B. Triggs, "Histograms of oriented gradients for human detection," in Proceedings - 2005 IEEE Computer Society Conference on Computer Vision and Pattern Recognition, CVPR 2005, 2005, vol. I, pp. 886-893, doi: 10.1109/CVPR.2005.177.

[3] H. Nam and B. Han, "Learning Multi-domain Convolutional Neural Networks for Visual Tracking," in Proceedings of the IEEE Computer Society Conference on Computer Vision and Pattern Recognition, 2016, vol. 2016-December, pp. 4293-4302, doi: 10.1109/CVPR.2016.465.

[4] C. Ma, J. B. Huang, X. K. Yang, and M. H. Yang, "Hierarchical Convolutional Features for Visual Tracking," IEEE International Conference on Computer Vision. Santiago, CHILE, pp. 3074-3082, 2015, doi: 10.1109/iccv.2015.352.

[5] M. Danelljan, G. Hager, F. S. Khan, and M. Felsberg, "Convolutional Features for Correlation Filter Based Visual Tracking," in Proceedings of the IEEE International Conference on Computer Vision, 2016, vol. 2016-February, pp. 621-629, doi: 10.1109/ICCVW.2015.84.

[6] M. Danelljan, A. Robinson, F. S. Khan, and M. Felsberg, "Beyond correlation filters: Learning continuous convolution operators for visual tracking," in ECCV 2016, 2016, vol. 9909 LNCS, pp. 472-488, doi: 10.1007/978-3-319-46454-1_29.

[7] R. Tao, E. Gavves, and A. W. M. Smeulders, "Siamese instance search for tracking," in Proceedings of the IEEE Computer Society Conference on Computer Vision and Pattern Recognition, 2016, vol. 2016-December, pp. 1420-1429, doi: 10.1109/CVPR.2016.158.

[8] L. Bertinetto, J. Valmadre, J. F. Henriques, A. Vedaldi, and P. H. S. Torr, "Fully-Convolutional Siamese
Networks for Object Tracking," ECCV 2016 Workshops, vol. 9914, pp. 850-865, 2016, doi: 10.1007/978-3-31948881-3_56.

[9] D. Held, S. Thrun, and S. Savarese, "Learning to track at 100 FPS with deep regression networks," in Computer Vision - ECCV 2016. Lecture Notes in Computer Science, 2016, vol. 9905 LNCS, doi: 10.1007/978-3319-46448-0_45.

[10] S. Yun, J. Choi, Y. Yoo, K. Yun, and J. Y. Choi, "Action-Decision Networks for Visual Tracking with Deep Reinforcement Learning," 30th IEEE/CVF Conference on Computer Vision and Pattern Recognition (CVPR). pp. 1349-1358, 2017, doi: 10.1109/cvpr.2017.148.

[11] L. Yang, R. Liu, D. Zhang, and L. Zhang, "Deep Location-Specific Tracking," in Proceedings of the 25th ACM International Conference on Multimedia, 2017, pp. 1309-1317, doi: 10.1145/3123266.3123381.

[12] A. H. A. El-Shafie, M. Zaki, and S. E. D. Habib, "Fast CNN-based object tracking using localization layers and deep features interpolation," in 15th International Wireless Communications and Mobile Computing Conference, IWCMC 2019, 2019, doi: 10.1109/IWCMC.2019.8766466.

[13] A. H. A. El-Shafie, M. Zaki, and S. E. D. Habib, "Towards an Efficient Hardware Implementation of CNN-Based Object Trackers," accepted in IEEE International Symposium on Circuits and Systems ISCAS-2021, 22-28 May 2021, Deagu, Korea.

[14] D. Han, J. Lee, J. Lee, and H. J. Yoo, "A Low-Power Deep Neural Network Online Learning Processor for Real-Time Object Tracking Application," IEEE Transactions on Circuits and Systems I: Regular Papers, 2019, doi: 10.1109/TCSI.2018.2880363.

[15] K. Chatfield, K. Simonyan, A. Vedaldi, and A. Zisserman, "Return of the Devil in the Details: Delving Deep into Convolutional Nets," in British Machine Vision Conference, 2014.

[16] Y. Wu, J. Lim, and M. H. Yang, "Object Tracking Benchmark," IEEE Transactions on Pattern Analysis and Machine Intelligence, vol. 37, no. 9, pp. 1834-1848, 2015, doi: 10.1109/tpami.2014.2388226.

[17] D. Przewlocka, M. Wasala, H. Szolc, K. Blachut, and T. Kryjak, "Optimisation of a Siamese Neural Network for Real-Time Energy Efficient Object Tracking," arXiv preprint arXiv:2007.00491, 2020.

[18] M. Wang, S. Lu, D. Zhu, J. Lin, and Z. Wang, "A HighSpeed and Low-Complexity Architecture for Softmax Function in Deep Learning," in IEEE Asia Pacific Conference on Circuits and Systems, APCCAS 2018, 2019, doi: 10.1109/APCCAS.2018.8605654.

[19] I. Kouretas and V. Paliouras, "Simplified Hardware Implementation of the Softmax Activation Function," in 8th International Conference on Modern Circuits and Systems Technologies, MOCAST 2019, 2019, doi: 10.1109/MOCAST.2019.8741677.

[20] "VC709 Evaluation Board for the Virtex-7 FPGA."

[Online]. Available:

https://www.xilinx.com/support/documentation/boards_a nd_kits/vc709/ug887-vc709-eval-board-v7-fpga.pdf. 
[21] "7 Series FPGAs Data Sheet: Overview." [Online]. Available: https://www.xilinx.com/support/documentation/data_she ets/ds180_7Series_Overview.pdf.

[22] R. V. Wicaksana Putra, M. Abdullah Hanif, and M. Shafique, "DRMap: A generic DRAM data mapping policy for energy-efficient processing of convolutional neural networks," in Proceedings - Design Automation Conference, 2020, doi: 10.1109/DAC18072.2020.9218672.

[23] D. Kim, J. Kung, and S. Mukhopadhyay, "A poweraware digital multilayer perceptron accelerator with onchip training based on approximate computing," IEEE Transactions on Emerging Topics in Computing, 2017, doi: 10.1109/TETC.2017.2673548.

[24] Q. Liu, J. Liu, R. Sang, J. Li, T. Zhang, and Q. Zhang, "Fast neural network training on FPGA using quasiNewton optimization method," IEEE Transactions on Very Large Scale Integration (VLSI) Systems, 2018, doi: 10.1109/TVLSI.2018.2820016.

[25] D. Shin, J. Lee, J. Lee, and H. J. Yoo, "DNPU: An 8.1TOPS/W reconfigurable CNN-RNN processor for general-purpose deep neural networks," in Digest of Technical Papers - IEEE International Solid-State Circuits Conference, 2017, doi: 10.1109/ISSCC.2017.7870350. 